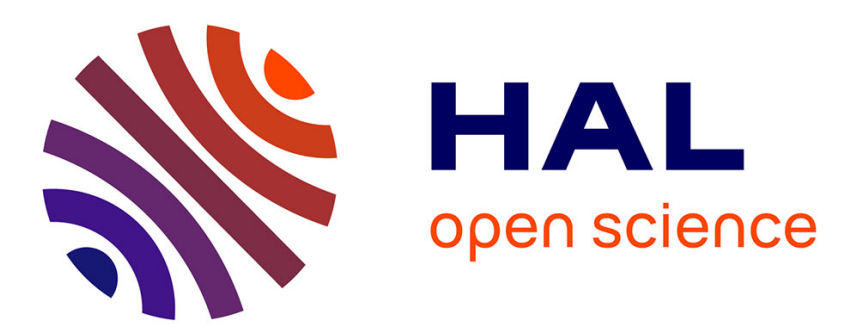

\title{
A simple derivation of the bulk strain field - MISFIT dislocation equilibrium in semiconductor single heterostructures
}

\author{
A. Fortini, M. Brault
}

\section{- To cite this version:}

A. Fortini, M. Brault. A simple derivation of the bulk strain field - MISFIT dislocation equilibrium in semiconductor single heterostructures. Revue de Physique Appliquée, 1990, 25 (11), pp.1037-1047. 10.1051/rphysap:0199000250110103700 . jpa-00246275

\section{HAL Id: jpa-00246275 https://hal.science/jpa-00246275}

Submitted on 1 Jan 1990

HAL is a multi-disciplinary open access archive for the deposit and dissemination of scientific research documents, whether they are published or not. The documents may come from teaching and research institutions in France or abroad, or from public or private research centers.
L'archive ouverte pluridisciplinaire HAL, est destinée au dépôt et à la diffusion de documents scientifiques de niveau recherche, publiés ou non, émanant des établissements d'enseignement et de recherche français ou étrangers, des laboratoires publics ou privés. 


\title{
REVUE DE PHYSIQUE APPLIQUÉE
}

Revue Phys. Appl. 25 (1990) 1037-1047

NOVEMBRE 1990, PAGE 1037

Classification

Physics Abstracts

$61.70 \mathrm{G}-73.40 \mathrm{~L}$

\section{A simple derivation of the bulk strain field - MISFIT dislocation equilibrium in semiconductor single heterostructures}

\author{
A. Fortini and M. Brault \\ Université de Caen-ISMRa, LERMAT, URA CNRS 1417, 14032 Caen Cedex, France
}

(Received 23 March 1990, revised 18 July 1990, accepted 7 August 1990)

\begin{abstract}
Résumé. - On calcule l'équilibre thermodynamique entre le champ de contraintes interne et les dislocations d'interface d'une hétérostructure à semiconducteurs, dans un modèle à deux dimensions qui permet une résolution complète, grâce à un choix simple et physique de conditions aux limites. Le modèle révèle nettement une transition de phase à un désaccord critique de maille, ou une épaisseur critique de la couche épitaxiale, et permet de séparer de l'énergie élastique, la contribution du cœur qui est ensuite laissée comme paramètre. Une comparaison soignée avec les théories de van der Merwe et de Matthews et Blakeslee permet de conclure que toutes trois conduisent à des résultats presque identiques. Par suite, l'accord actuellement reconnu, d'un bon nombre de résultats expérimentaux avec la théorie de Matthews concerne en fait toutes les théories d'équilibre et, en particulier, la théorie proposée. Il semble généralement admis, d'autre part, que les écarts importants qui demeurent sont imputables à des comportements métastables au cours de la croissance.
\end{abstract}

\begin{abstract}
The thermodynamic equilibrium between bulk strain field and interface dislocations in lattice mismatched semiconducting single heterostructures, is calculated in a completely solvable two-dimensional model, thanks to a choice of simple physical boundary conditions. The model clearly exhibits a phase transition at a critical misfit or a critical thickness of the epilayer, and allows to separate out from the elastic energy, the core contribution which is left, next, as a parameter. Through a carefull comparison with van der Merwe's and Matthews and Blakeslee's approaches, it is found that all of them turn out to lead to almost identical results. It follows that the widespread agreement of Matthews' theory with number of experimental data holds for all equilibrium theories, and in particular, the present one, as well. The remaining strong discrepancies, on the other hand, are admittedly ascribed to metastable behaviour in growing processes.
\end{abstract}

\section{Introduction.}

The release of strain by misfit dislocations in lattice misma c e semicon uc ing e eros ructures is a topic of considerable technical importance, mainly because the epilayers quality is severely degraded by interface defects. Moreover, this topic has recently received an increasing attention in connection with the development of superlattices $[1,2]$ and the emerging technology of the heteroepitaxy of GaAs on $\mathrm{Si}$ substrates [3-5].

The lattice misfit is well known to be first accomodated by a bulk strain field, up to a critical thickness of the epilayer, the knowledge of which is essential in device fabrication. The configuration of misfit dislocations which sets in at critical thickness, was first investigated by van der Merwe [6-8] in terms of minimum energy principle. Calculations based on various assumptions about interfacial forces lead to the equilibrium values of the dislocation density and the critical thickness through rather elaborated mathematics and computations. An alternative equilibrium model based on a balance between forces acting on dislocations, has been proposed by Matthews and Blakeslee [9]. Also, dynamical theories exist, taking explicitly into account nucleation and propagation processes of dislocations [10-13].

The purpose of the present paper is to show that, by means of a suitable choice of boundary con- 


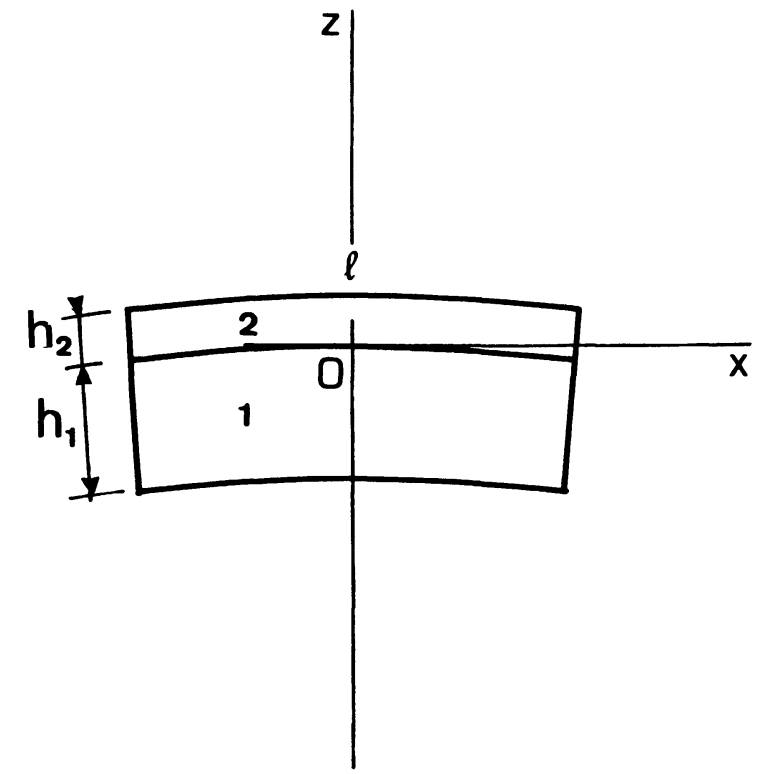

Fig. 1. - Geometric configuration of the two-dimensional scheme used in the analysis of elastic and plastic deformation of the single heterostructure.

ditions, a fairly simplified derivation of the equilibrium case can be obtained, which clearly exhibits a critical misfit transition. We shall consider a simple heterostructure fabricated by an overgrowth of a thin layer 2 of thickness $h_{2}$ onto a substrate 1 of thickness $h_{1}$ (Fig. 1). The lattice constants, $a_{1}$, $a_{2}$ of the constituting materials are not identical, the misfit being characterized by the parameter

$$
\boldsymbol{\beta}=\left(a_{2}-a_{1}\right) / a_{1} .
$$

Above a critical value $h_{\mathrm{c}}$ of $h_{2}$, the misfit accomodation is shared between a bulk strain field giving rise to an overall bending of the structure, and a plastic deformation due to a more or less regular grid of dislocations located in the interface. If $\beta_{\mathrm{s}}$ and $\beta_{\mathrm{d}}$ denote these respective contributions, we thus have

$$
\beta=\beta_{\mathrm{s}}+\beta_{\mathrm{d}} .
$$

For the sake of simplicity, calculations will be restricted to the thin-epilayer-thick-substrate case, and edge dislocations in a simple cubic lattice will be assumed. In the actual physical situation, the misfit dislocations have been identified as being mainly of $60^{\circ}$ type. However, on account of the degree of agreement with experiment usually expected from this kind of theoretical predictions (see the discussion below), together with unavoidable approximations (e.g. in the estimate of the core contribution), crystallographic details can be simplified without further loss of precision. For the same reasons, it will be sufficient to consider, consistently, the bulk strain and the plastic deformation along only one the two interface dimensions, say $x$, and the perpendicular $z$ dimension. Indeed, we are mainly interested in the balance between elastic and plastic relaxation, and to the degree of approximation used here, the contributions of each dimension of the dislocation grid simply add up. The mathematical description will thus be restricted to a two dimensions scheme in the $x z$-plane, assuming uniformity in the third $y$ dimension (Fig. 1).

Calculations of the bulk strain field associated with the strain $\beta_{s}$ have been already published [14, 15]. Let us briefly recall that in our simplified two dimensional single heterostructure of transverse dimension $\ell$ (Fig. 1), if equal and isotropic elastic constants for both materials are assumed, the elastic energy per unit length in the $y$ dimension is given by

$$
W_{\mathrm{s}}=\frac{\ell h_{1} h_{2}\left(h_{1}^{3}+h_{2}^{3}\right)}{2\left(h_{1}+h_{2}\right)^{4}} E \beta_{\mathrm{s}}^{2},
$$

where $E$ is the Young modulus. In the case of a thin epilayer on a thick substrate $\left(h_{2} \gg h_{1}\right)$ :

$$
W_{\mathrm{s}} \cong \frac{1}{2} \ell h_{2} E \beta_{\mathrm{s}}^{2} \text {. }
$$

The stress field associated with misfit dislocations is calculated in the next section, by combining our choice of boundary conditions with the stress function method $[6,16]$. In section 3 the resulting expression of the total mechanical energy is obtained through addition of both contributions, and leads to the equilibrium value of $\beta_{\mathrm{d}}$. The core energy which will be first treated as purely elastic for mathematical convenience, will then be separated out, and left as a parameter, on account of the lack of quantitative information thereon. Section 4 is devoted to a comparison with other approaches and a discussion on the reliability of equilibrium methods with regard to available experimental data.

\section{Interfacial energy of misfit dislocations.}

Since elastic and plastic deformations, and the related energies, will be additively superimposed, they can be separately considered. Thus, in this section, all quantities will be regarded, without any confusion, as pertaining to the plastic case alone.

In our two-dimensional approximation of simple cubic structures, we shall consider a regular array of parallel edge dislocations, lying along the $y$ direction, and spaced by a distance $p$ along the $x$-axis. The atomic configuration in the period $-\frac{1}{2} p \leqslant x \leqslant \frac{1}{2} p$ of the $x-z$ plane is schematically represented in figure 2. The number of cells along the $x$-axis in the crystal 1 is larger by one than that of the crystal 2 . Because of the exponential decrease of strain with increasing distance from the interface plane $(z=0)$, the crystal extending on either side will be takèn as 


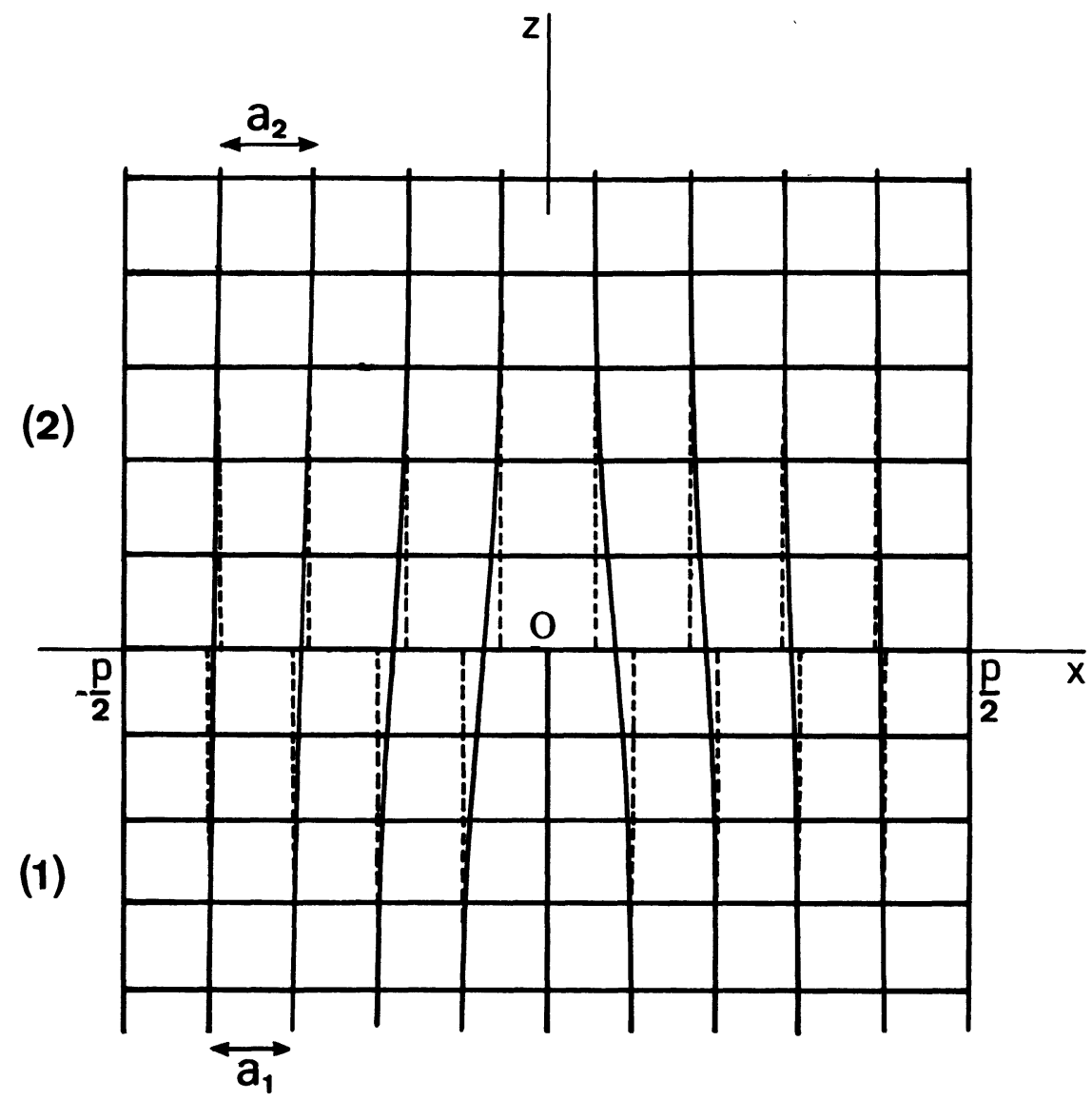

Fig. 2. - Sketch of the lattice distortion in a period $\left(-\frac{1}{2} p \leqslant x \leqslant+\frac{1}{2} p\right)$, due to a dislocation array, showing the linear displacement of the atoms at the interface, relative to their ideal positions.

infinite. The validity of this approximation will be checked below in a self-consistent way.

At a distance of order $p / 2$ from the interface the crystal ordering is recovered and, therefore, the true values $a_{1}, a_{2}$ of the lattice constants too. Taken the origin of coordinates at the trace of the dislocation axis in the $x-z$ plane, the projection of the ideal lattice nodes on the $x$-axis (as indicated by the dotted lines in Fig. 2) are symmetrically positioned. On the right-hand side of the dislocation $(x \geqslant 0)$, in the crystal 1 and 2 , respectively, their abscissa are given by

$$
x_{n}^{(1)}=n a_{1} \text { and } x_{n}^{(2)}=\left(n-\frac{1}{2}\right) a_{2},
$$

with $n$ integer. The true positions $x_{n}$ of the atoms, at equilibrium are located between $x_{n}^{(1)}$ and $x_{n}^{(2)}$. Since, in addition, elastic constants are taken equal in both materials, the $x_{n}$ 's just fall at the middle point, except in the strongly distorded core region, i.e. for $x \geqslant 0$,

$$
x_{n}=\frac{1}{2}\left[n\left(a_{1}+a_{2}\right)-\frac{1}{2} a_{2}\right] \text {. }
$$

(A some more elaborated expression would hold in the case of distinct elastic constants in 1 and 2). As a result, the displacements at the $n$-th site on the right, along the interface, can now be written as

$$
\left(\begin{array}{l}
u_{n x}^{(2)} \\
u_{n x}^{(1)}
\end{array}\right)=\mp \frac{1}{2} n\left(a_{2}-a_{1}\right) \pm \frac{1}{4} a_{2}
$$

In the assumption of elastic continuum underlying the present treatment, this leads us to interpolate the displacement $u_{x}(x, 0)$ along the interface, in the range,${ }_{2} p$ ut outsi e $\mathrm{t}$ e core, y t e o owing linear functions

$$
\begin{aligned}
\left(\begin{array}{l}
u_{x}^{(2)}(x, 0) \\
u_{x}^{(1)}(x, 0)
\end{array}\right)=\mp \frac{a_{2}-a_{1}}{a_{2}+a_{1}} x \pm & \frac{a_{2} a_{1}}{2\left(a_{2}+a_{1}\right)} \\
& \cong \mp \frac{1}{2} \beta_{\mathrm{d}} x \pm \frac{1}{4} a,
\end{aligned}
$$

A similar symmetrical function holds in the range $\left(-\frac{1}{2} p, 0\right)$. The dislocation misfit parameter $\beta_{d}$ has 


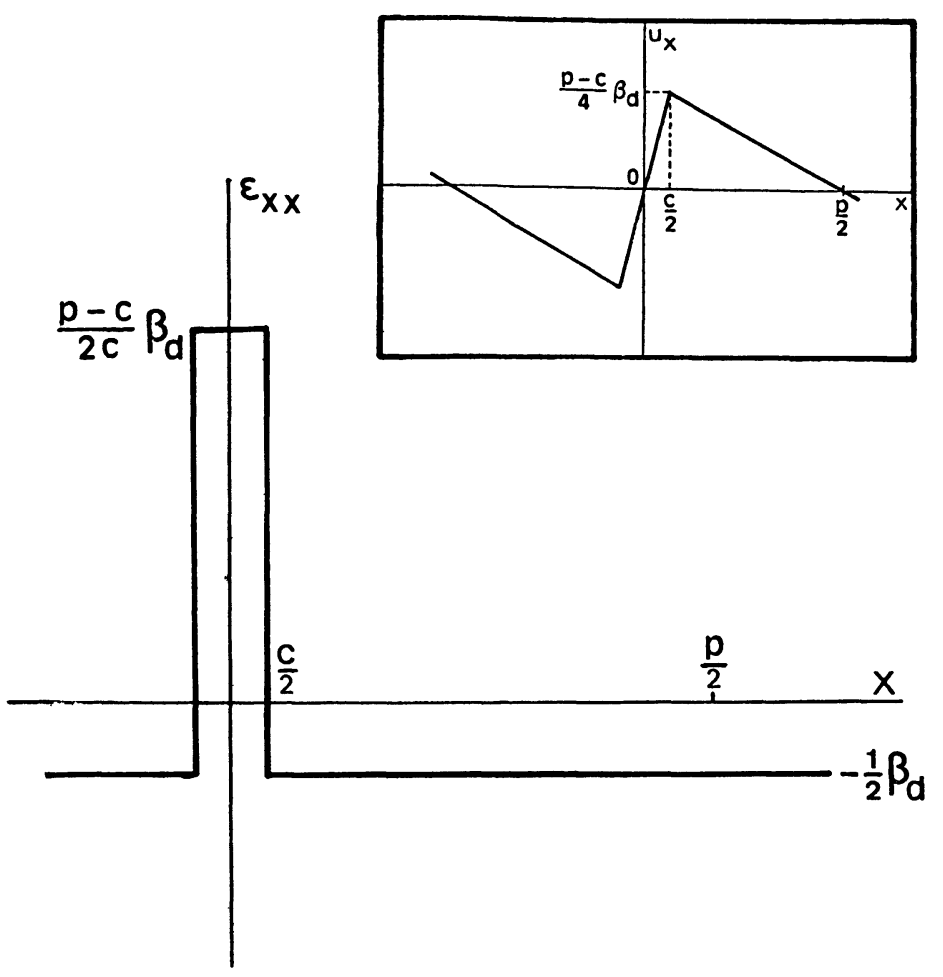

Fig. 3. - Interface strain profile in the upper half-crystal of figure 2, derived from the displacement function shown in the inset. The unphysical elastic behaviour assumed in the core $\left(-\frac{1}{2} c \leqslant x \leqslant \frac{1}{2} c\right)$ is separated out by analytical means.

been introduced, together with an average value $a$ of $a_{1}$ and $a_{2}$.

Inside the core, which will be assumed to extend over a width $c$, the displacement will be described, for the time being, by means of a simple linear interpolation (Fig. 3) giving

$$
\left(\begin{array}{l}
u_{x}^{(2)} \\
u_{x}^{(1)}
\end{array}\right)= \pm\left(\frac{a}{c}-\beta_{\mathrm{d}}\right) \frac{x}{2}, \quad\left(-\frac{1}{2} c \leqslant x \leqslant \frac{1}{2} c\right) \text {. }
$$

This procedure has the advantage of preserving everywhere the continuity of the displacement $u_{x}(x, 0)$ which is required in the mathematical treatment below. The counterpart is that a spurious unphysical contribution is introduced in the interfacial energy, which we shall have to separate out later on.

Next, if one assumes the displacement $u(x, z)$ sufficiently regular near $z=0$, the boundary strain component are easily deduced from (4) and (5) by

$$
\varepsilon_{x x}(x, 0)=\left(\frac{\partial u_{x}}{\partial x}\right)_{z=0}=\frac{\mathrm{d}}{\mathrm{d} x} u_{x}(x, 0) .
$$

This leads to the strain profiles shown in figure 3 , for the upper half-crystal. Notice that, as $u_{x}(x, 0)$ vanishes at the ends of the range $\left(-\frac{1}{2} p, \frac{1}{2} p\right)$, necessarily the mean value of $\varepsilon_{x x}(x, 0)$ is zero.
2.1 STRESS FIELD OF MISFIT DISLOCATIONS. - We now turn to the calculation of the two dimensional stress field, assuming isotropic medium. This field is periodic, with a period $p$, in the $x$ direction, and vanishes away from the interface, i.e. for $z= \pm \infty$. Following van der Merwe [6], we start with the stress function [16] $\Phi(x, z)$ satisfying the compatibility equation, $\nabla^{4} \Phi(x, z)=0$. The $n$-th Fourier component of the suited general solutions is given by

$$
\begin{aligned}
\Phi_{n}(x, z)=\left(B_{n}-A_{n} z\right) \exp (-2 & \pi n|z| / p) \times \\
& \times \exp (2 i \pi n x / p),
\end{aligned}
$$

where $A_{n}, B_{n}$ are constants. Putting for brevity $k_{n}=2 \pi n / p$, this leads to the following stress components, satisfying the boundary conditions $\sigma_{z z}(x, 0) \equiv 0$ and $\sigma_{x z}\left( \pm \frac{1}{2} p, 0\right)=0$

$$
\begin{aligned}
\sigma_{x x} & =\frac{\partial^{2} \Phi}{\partial z^{2}}= \pm \sum_{n=1}^{\infty} k_{n} A_{n}\left(2 \mp k_{n} z\right) \mathrm{e}^{\mp k_{n} z} \cos \left(k_{n} x\right) \\
\sigma_{z z} & =\frac{\partial^{2} \Phi}{\partial x^{2}}=\sum_{n=1}^{\infty} k_{n}^{2} A_{n} z \mathrm{e}^{\mp k_{n} z} \cos \left(k_{n} x\right) \\
\sigma_{x z} & =-\frac{\partial^{2} \Phi}{\partial x \partial z}= \\
& =-\sum_{n=1}^{\infty} k_{n} A_{n}\left(1 \mp k_{n} z\right) \mathrm{e}^{\mp k_{n} z} \sin \left(k_{n} x\right)
\end{aligned}
$$


The zero Fourier component is missing because the strain mean value vanishes as above mentioned. The upper sign refers to the crystal 2 acted upon by the crystal 1, and the opposite holds for the lower sign. From elementary Elasticity Theory [16], the strain components are given as function of stress by

$$
\begin{aligned}
& \varepsilon_{x x}=\frac{1}{2 G}\left[(1-\nu) \sigma_{x x}-\nu \sigma_{z z}\right] ; \\
& \varepsilon_{z z}=\frac{1}{2 G}\left[(1-\nu) \sigma_{z z}-\nu \sigma_{x x}\right] ; \\
& \varepsilon_{x z}=\frac{\sigma_{x z}}{G} .
\end{aligned}
$$

$G$ denotes the shear modulus and $\nu$ the Poisson ratio. Using equations (6) we find, in particular, in the upper half-crystal, close to the interface,

$$
\varepsilon_{x x}(x, 0)=\sum_{n=1}^{\infty} \frac{1-\nu}{G} k_{n} A_{n} \cos \left(k_{n} x\right) .
$$

On applying this expansion to the profile of figure 3, we straigthforwardly obtain the Fourier coefficients

$$
A_{n}=\frac{G a p \sin (\pi n c / p)}{2 \pi^{2}(1-\nu) n^{2} c}
$$

which makes complete the determination of the stress components (6).

2.2 Stress ENERGY. - The stress energy of the dislocation array is defined as the energy required to change each half-crystal from the ideal configuration (dotted lines in Fig. 1) into the final distorded one. Using (7), we have in a period, per unit length in the $y$ direction

$$
\begin{aligned}
W_{\mathrm{p}}= & \frac{1}{2} \int_{-\infty}^{\infty} \mathrm{d} z \int_{-p / 2}^{p / 2}\left(\sigma_{x x} \varepsilon_{x x}+\sigma_{z z} \varepsilon_{z z}+\sigma_{x z} \varepsilon_{x z}\right) \mathrm{d} x \\
= & \frac{1}{2 G} \int_{-\infty}^{\infty} \mathrm{d} z \int_{0}^{p / 2} \cdot\left[(1-\nu)\left(\sigma_{x x}^{2}+\sigma_{z z}^{2}\right)\right. \\
& \left.-2 \nu \sigma_{x x} \sigma_{z z}+2 \sigma_{x z}^{2}\right] \mathrm{d} x
\end{aligned}
$$

The integration only requires the zero Fourier components of squares and products of stress components appearing in (9), which are given in turn, by summin over $n$ the related s uares and ro the Fourier components of same order in expansions (6). On performing all of these quite elementary calculations, we find

$$
W_{\mathrm{p}}=\frac{\pi(1-\nu)}{G} \sum_{n=1}^{\infty} n A_{n}^{2},
$$

in each half-crystal. Substituting next $A_{n}$ from (8), we obtain

$$
W_{\mathrm{p}}=\frac{G p^{2} a^{2}}{4 \pi^{3}(1-\nu) c^{2}} \sum_{n=1}^{\infty} \frac{\sin ^{2}(\pi n c / p)}{n^{3}} .
$$

The numerical series in the right-hand side is calculated in Appendix I as an expansion to increasing orders of $c / p$. For our purpose the leading term will be sufficient and yields the following expression of the interfacial energy per unit length along the $y$-axis

$$
W_{\mathrm{d}}=\frac{G l a^{2}}{4 \pi(1-\nu) p}\left(\ln \frac{p}{2 \pi c}+\frac{3}{2}\right) .
$$

\section{Thermodynamic equilibrium, critical misfit and critical thickness.}

3.1 SePARATION OF THE CORE ENERGY. - Before writing down the complete mechanical energy including at once the contributions of strain and dislocations, we have to separate out the unphysical " elastic » core energy initially introduced. The occurrence of such a spurious contribution was often discussed previously [8, 17], and generally neglected. An evaluation is possible, here, by taking both integrations of expression (9) in the range $(-c / 2$, $+c / 2)$

$$
\begin{array}{rl}
W_{\mathrm{c}}^{(\mathrm{el})}=\frac{1}{G} \int_{0}^{\mathrm{c} / 2} \mathrm{~d} z \int_{0}^{\mathrm{c} / 2} & \mathrm{~d} x\left[(1-\nu)\left(\sigma_{x x}^{2}+\sigma_{z z}^{2}\right)-\right. \\
& \left.-2 \nu \sigma_{x x} \sigma_{z z}+2 \sigma_{x z}^{2}\right]
\end{array}
$$

Since $p \gg c$, it will be sufficient to replace the stress components by the leading term of their expansions for $x \rightarrow 0$, and because of the continuity at the origin, these can be obtained through derivation by $x$ of the Fourier series (6). To lowest order in $c / p$, we obtain

$$
\begin{aligned}
& \sigma_{x x}= \pm \sum_{n=1}^{\infty} k_{n} A_{n}\left(2 \mp k_{n} z\right) \mathrm{e}^{-k_{n} z} \\
& \sigma_{z z}=\sum_{n=1}^{\infty} k_{n}^{2} A_{n} z \mathrm{e}^{\mp k_{n} z} \\
& \sigma_{x z}=-\sum_{n=1}^{\infty} k_{n}^{2} A_{n}\left(1 \mp k_{n} z\right) \mathrm{e}^{\mp k_{n} z} x .
\end{aligned}
$$

On substituting again $A_{n}$ from (8), these components are found to be expressed in terms of the following series and its derivatives with respect to $z$

$$
S(z)=\sum_{n=1} \frac{\text { si } \frac{1}{-}}{n} \mathrm{e}^{-k_{n} z}=\operatorname{Arctg} \frac{c}{2 z},
$$

for $z \geqslant 0$. Details of calculations are given in Appendix II. Hence, the stress components (12) pertaining to the upper half-crystal

$$
\begin{aligned}
& \sigma_{x x}=-\frac{G}{1-\nu} \frac{2 a}{\pi c}\left(S+\frac{1}{2} z S^{\prime}\right) ; \\
& \sigma_{z z}=\frac{G}{1-\nu} \frac{a z S^{\prime}}{\pi c} ; \\
& \sigma_{x z}=\frac{G}{1-\nu} \frac{a x}{\pi c}\left(S^{\prime}+z S^{\prime \prime}\right) .
\end{aligned}
$$


On substituting into equation (11), one finds after some integrations (see Appendix II), the value of $W_{\mathrm{c}}^{(\mathrm{el})}$ to zero order in $c / p$, allowing us to write

$$
W_{\mathrm{c}}^{(\mathrm{el})}=W_{\mathrm{c}}^{(\mathrm{el})}(0)+W_{\mathrm{c}}^{(\mathrm{el})}(c)
$$

where $W_{\mathrm{c}}^{(\mathrm{el})}(c)$ is a small $c$-dependent correction and

$$
W_{\mathrm{c}}^{(\mathrm{el})}(0)=\frac{G a^{2}}{4 \pi(1-\nu)}\left(1.696-\frac{0.289}{1-\nu}\right) .
$$

Now, the true energy of the matter in the range $\left(-\frac{1}{2} c, \frac{1}{2} c\right)$ including the strongly distorded region, say $\Gamma(c)$ in units of $G a^{2} / 4 \pi(1-\nu)$, must be substituted in expression (10). For convenience the resulting expression of $W_{\mathrm{d}}$ will be rewritten as

$$
\begin{aligned}
& W_{\mathrm{d}}=\frac{G \ell a^{2}}{4 \pi(1-\nu) p} {\left[\ln \frac{p}{a}-\ln 2 \pi+\frac{3}{2}+\right.} \\
&\left.\quad+\ln \frac{a}{c}-W_{\mathrm{c}}^{(\mathrm{el})}(0)-W_{\mathrm{c}}^{(\mathrm{el})}(c)+\Gamma(c)\right] .
\end{aligned}
$$

Unfortunately, the core energy is sensitive to the actual atomic structure and particularly to the possible reconstruction of dangling bonds, which are not well established up to now. It follows that no reliable

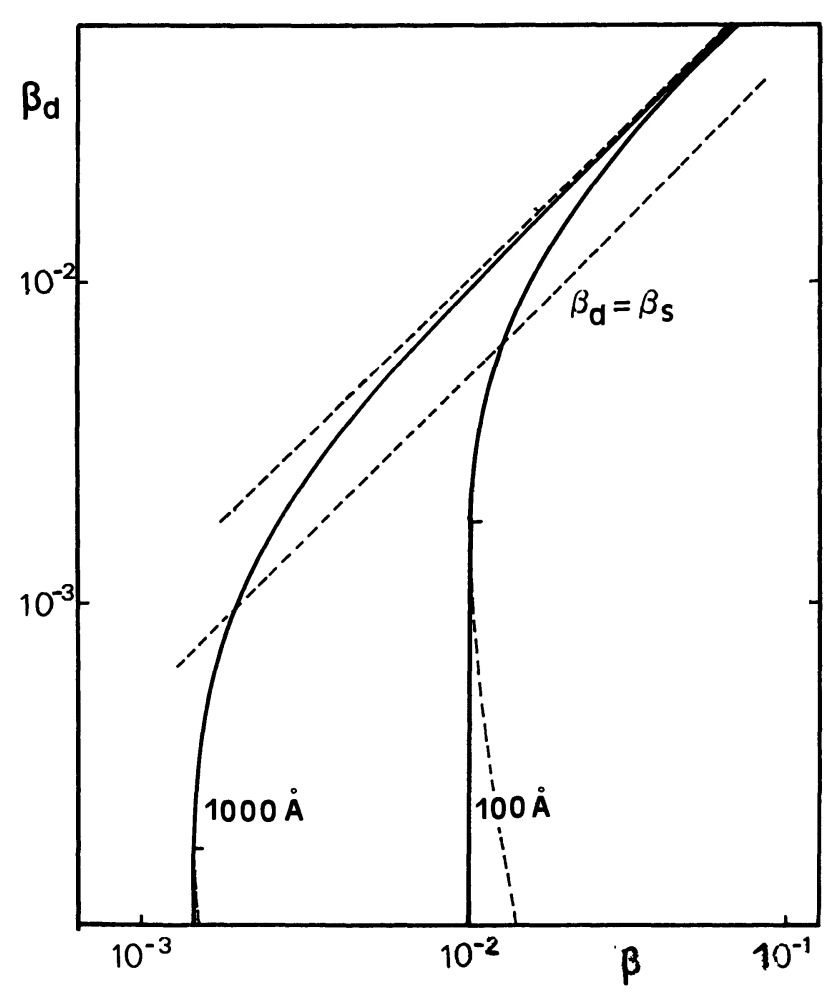

Fig. 4. - Dislocation part $\beta_{\mathrm{d}}$ to the misfit accomodation at equilibrium as a function of the constituent misfit $\beta$, for two selected values of the layer thickness $(100 \AA$ and $1000 \AA$ ). The phase-transition-like behaviour is well exhibited by the quick decrease occuring at critical misfit. The straight line $\beta_{\mathrm{d}}=\beta_{\mathrm{s}}=\frac{1}{2} \beta$ is the locus of the transition points, according to the present definition. value is available and, therefore, we shall restrict ourselves to rewrite the interfacial energy with the help of a suitable core energy parameter $\gamma$. To this end we notice that even though the choice of $c$ is somewhat arbitrary, an exact expression of $W_{\mathrm{d}}$ cannot depend on it. This means that provided $c$ is large enough to overlap the elastic region, all $c$ dependent terms in the square bracket above combine so as to eliminate the parameter $c$. Thus, $\gamma$ will be most properly defined by the $c$-independent quantity

$$
\gamma=\Gamma(c)+\ln \frac{a}{c}-W_{\mathrm{c}}^{(\mathrm{el})}(c)
$$

leading to

$$
\begin{aligned}
W_{\mathrm{d}}=\frac{G l a^{2}}{4 \pi(1-\nu) p} \times \\
\times\left[\ln \frac{p}{a}-2.034+\frac{0.289}{1-\nu}+\gamma\right] .
\end{aligned}
$$

From existing estimates of the energy of dangling bonds, distorted and possibly reconstructed bonds, based on suited atomic potentials [18], an order of $1 \mathrm{eV}$ per site is quite probable, leading to values of $\gamma$ of about 1 in usual semiconducting compounds. This is also close to the current estimate of the Peierls core parameter [17]. It can perhaps be regarded as small but not negligible, as early pointed out by van der Merwe [8] and, recently, by Eaglesham et al. [12]. So, it must be born in mind in the comparison of theory and experiment.

\subsection{TOTAL ENERGY AND THERMODYNAMIC EQUILI-} BRIUM. - The total mechanical energy of the $\left(\boldsymbol{\beta}_{\mathrm{s}}, \boldsymbol{\beta}_{\mathrm{d}}\right)$ configuration is now obtained by adding up (3) and (13)

$$
\begin{aligned}
W=\frac{1}{2} \ell h_{2} E \beta_{\mathrm{s}}^{2}+ & \frac{G \ell a^{2}}{4 \pi(1-\nu) p} \times \\
& \times\left[\ln \frac{p}{a}-2.034+\frac{0.289}{1-\nu}+\gamma\right] .
\end{aligned}
$$

It will be convenient to rewrite this expression in units of $\frac{1}{2} \ell h_{2} E$. Taking the relation $E / 2 G=1+\nu$ into account and recalling that $a / p=\beta_{\mathrm{d}}$, we obtain

$$
\begin{aligned}
w=\left(\beta-\beta_{\mathrm{d}}\right)^{2}+\left(2 \alpha \beta_{\mathrm{d}} / h_{2}\right) \times & \\
\times & {\left[-\ln \beta_{\mathrm{d}}-2.034+0.289 /(1-\nu)+\gamma\right], }
\end{aligned}
$$

with $\alpha=a / 8 \pi\left(1-\nu^{2}\right)$. A minimum of $w$ occurs for $\beta_{\mathrm{d}}$ satisfying the equation

$$
\begin{array}{r}
\beta=\beta_{\mathrm{d}}+\left(\alpha / h_{2}\right)\left[-\ln \beta_{\mathrm{d}}-3.034+0.289 /\right. \\
(1-\nu)+\gamma] .
\end{array}
$$

The function $\beta\left(\beta_{\mathrm{d}}\right)$ has in turn a minimum (see Fig. 4) for $\beta_{\mathrm{d}}=\alpha / h_{2}$. It follows that given $\beta$ above the minimum, two roots for $\beta_{\mathrm{d}}$ are obtained, the 
largest one of which only corresponds to a minimum of the energy, as it can be readily checked by calculating the second derivative $\mathrm{d}^{2} w / \mathrm{d} \beta_{\mathrm{d}}^{2}$. No thermodynamic equilibrium can exist below the minimum so that the related value of $\beta$ satisfying the following equation

$$
\begin{array}{r}
\beta_{\mathrm{c}}=\left(\alpha / h_{2}\right)\left[-\ln \left(\alpha / h_{2}\right)-2.034+0.289 /\right. \\
(1-\nu)+\gamma]
\end{array}
$$

defines the critical misfit $\beta_{\mathrm{c}}$.

It is more convenient to consider the inverse function $\beta_{\mathrm{d}}(\beta)$ which, from the foregoing, must be drawn as shown by the full lines in figure 4 , to be physically significant. It is worth noticing its phasetransition-like step behaviour : below $\beta_{c}$, we have a range of coherence in which the misfit $\beta$ is accomodated by the bulk strain field alone, whilst beyond $\beta_{\mathrm{c}}$, a state is quickly reached in which $\beta$ is completely accomodated by dislocations.

Now, if $\beta$ is regarded as a constituent parameter, equation (15) defines a critical value of the epilayer thickness $h_{2}$. The natural definition of the threshold as $h_{2}$ is increased is then obtained by setting $\boldsymbol{\beta}_{\mathrm{c}}=\boldsymbol{\beta}$. In practice, however, the measured transition point is somewhat dependent upon the experimental methods employed. These mainly rely on the observation of an abrupt change in some physical property : reduction of the tetragonal lattice distortion measured by X-ray diffraction [19-22], Rutherford back scattering and ion channeling [23] ; strain induced shift of the photoluminescence peak together with the related line broadening and drop of quantum efficiency [24-28] ; direct appearence of the dislocation patterns by electron microscopy [11, 23, 26, 29], electron-beam-induced current [30], changes in surface morphology [28], transport properties [31, 32]... Because of this large variety of methods a suited definition ought to be adopted in each case. For the need of the present analysis, it will be most reasonable to define the transition as the point where the misfit is equally shared between strain and dislocations, say $\beta_{\mathrm{d}}=\beta_{\mathrm{s}}=\frac{1}{2} \beta$. This leads, from (14), to the following expression of the experimental critical width

$$
\begin{array}{r}
h_{\mathrm{c}}^{(\mathrm{ex})}=(2 \alpha / \beta)[-\ln \beta-1.341+0.289 / \\
(1-\nu)+\gamma] .
\end{array}
$$

Strictly speaking, this expression is only valid as far as $h_{\mathrm{c}}^{\text {(ex) }}$ falls beyond the limit for which the above approximation of semi-infinite crystals holds. From equations (6) the stress and strain components decrease mainly as $\exp (-2 \pi|z| / p)$. As $p=a / \beta_{\mathrm{d}}$, one easily obtains

$$
\begin{aligned}
2 \pi h_{\mathrm{c}}^{(\mathrm{ex})} / p=[-\ln \beta-1.341+0.289 / \\
(1-\nu)+\gamma] / 4\left(1-\nu^{2}\right),
\end{aligned}
$$

which will be found to amount to 1.9 at $\beta=10^{-3}$ and 1.3 at $\beta=10^{-2}$, with the numerical values taken below. Thus, due to the weak logarithmic dependence in $\beta$ of $2 \pi h_{\mathrm{c}}^{(\mathrm{ex})} / p$, the semi-infinite crystal approximation is acceptable up to $\beta$ values of a few $10^{-2}$.

\section{Comparison with other theories and experiment. Discussion.}

Assuming the core energy parameter equal to 1 , the critical thickness defined by equation (16), as a function of $\beta$, is smoothly sensitive to the material elastic constants (through the Poisson ratio), and so, $h_{\mathrm{c}}^{(\mathrm{ex})}(\beta)$ can be conveniently compared with other theoretical predictions and available experimental data, by assuming average values for the elastic constants and the lattice parameter $a$. In fact, the latter represents a slide distance, i.e. the magnitude of the Burger vector.

The van der Merwe theory is dominated, in the case of a two-dimensional semi-infinite overgrowth, by the following expression of the interfacial energy [6]

$$
\begin{aligned}
W_{\mathrm{d}}=\left(G l a / 4 \pi^{2}\right)[1 & +\gamma_{\mathrm{d}}-\sqrt{1+\gamma_{\mathrm{d}}^{2}}-\gamma_{\mathrm{d}} \times \\
& \left.\times \ln \left(2 \gamma_{\mathrm{d}} \sqrt{1+\gamma_{\mathrm{d}}^{2}}-2 \gamma_{\mathrm{d}}^{2}\right)\right] .
\end{aligned}
$$

Present notations have been used and $\gamma_{\mathrm{d}}=\pi \beta_{\mathrm{d}} /(1-\nu)$. On combining this contribution with the strain energy (3), and proceeding as before, a minimum occurs at

$$
\beta=\beta_{\mathrm{d}}-\left(\alpha / h_{2}\right) \ln \left(2 \gamma_{\mathrm{d}} \sqrt{1+\gamma_{\mathrm{d}}^{2}}-2 \gamma_{\mathrm{d}}^{2}\right) \text {. }
$$

Since $\gamma_{\mathrm{d}} \ll 1$, this expression will be simplified into

$$
\beta=\beta_{\mathrm{d}}-\left(\alpha / h_{2}\right) \ln \left[2 \pi \beta_{\mathrm{d}} /(1-\nu)\right]
$$

which is close to that given by Maree et al. [11]. The critical thickness, however, cannot properly be obtained by taking the value of $h_{2}$ in the limit $\beta_{\mathrm{s}}=\boldsymbol{\beta}$, as noticed by these authors, since in that limit the logarithm becomes divergent $\left(\beta_{d}=0\right)$. We notice, instead, that the van der Merwe expression (14), so we shall define the experimental transition threshold the same way as before, which leads to the following van der Merwe critical thickness

$h_{\mathrm{c}}^{(v \mathrm{M})}=(2 \alpha / \beta)(-\ln \beta-\ln [\pi /(1-\nu)])$.

This expression is almost identical with (16) from which it only differs, through the constant $\ln [\pi /(1-\nu)]$, by an amount of the order of the core contribution.

The theoretical law established by Matthews and 
Blakeslee [9], on the other hand, will be taken, in the simple heterostructure case, in the usual form

$h_{\mathrm{c}}^{(\mathrm{MB})}=[a / 4 \pi(1+\nu) \beta]\left[\ln \left(h_{\mathrm{c}}^{(\mathrm{MB})} / a\right)+1\right]$.

Also, it will be of interest to consider the People and Bean energy balance model in which [10]

$h_{\mathrm{c}}^{(\mathrm{PB})}=\left[a(1-\nu) / 32 \pi(1+\nu) \beta^{2}\right] \ln \left(h_{\mathrm{c}}^{(\mathrm{PB})} / a\right)$.

Now, the critical thickness, as given by expressions (16) and (18-20), is plotted versus the misfit $\beta$ in figure 5, with the typical values $a=4 \AA$ and $\nu=1 / 3$. Besides the similarity already mentioned between the van der Merwe and the present theoretical laws, it is worth noticing that the latters are, at once, very close to Matthews' law for single heterostructures to within the degree of uncertainty of all these analysis (they would be almost identical if the transition were defined from (15) with $\beta_{c}=\beta$ ).

For the sake of comparison with experiment, points obtained from various measurement methods in several heteroepitaxial systems have been plotted in figure 5. Some of them pertain to quantum well structures for which the related Matthews' law has been plotted, also. We first observe that, in spite of the spread which can likely be attributed to the limited accuracy of theoretical predictions, experimental detections, and our averaging procedure, a fairly large number of points are in satisfactory agreement with the theoretical laws. This is in accordance with the emerging belief that Matthews's law adequately predicts the critical layer thickness, as repeatedly pointed out in recent years $[20,25,28$,

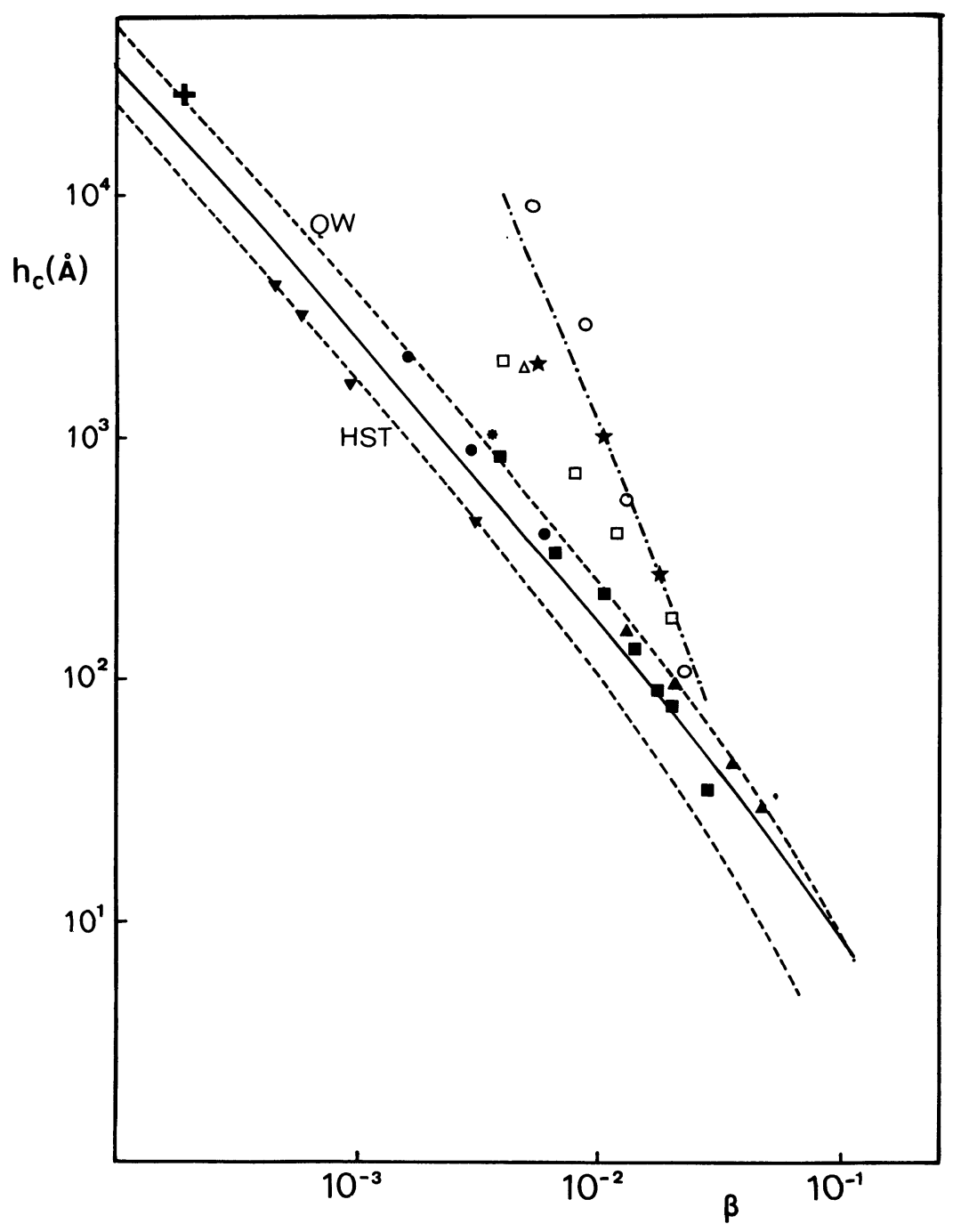

Fig. 5- Theoretical plots of the critical thickness $h_{\mathrm{c}}^{(\mathrm{ex})}$ as a function of the misfit $\beta$ : the present theory (full line) is very close to van der Merwe's; Matthews and Blakeslee (dashed lines) pertaining to single heterostructures (HST) and quantum wells (QW); People and Bean (dot-dashed line). Experimental points are extracted from references [19] (cross), 29 (closed circles), 23 (asterix), 20 (open circles), 22 (stars), 11 (open triangles), 25 (closed triangles), 30 (open squares), 28 (closed squares), 33 (inverted closed triangles). 
31-33]. The point is that, since all equilibrium laws are, from the present analysis, almost identical, this necessarily remains true for the other models derived from the minimum energy principle, at least in so far as some care is taken in the definition of the transition. It is worth noticing, in addition, that the agreement of the present theory with some set of experimental points is still improved by choosing a larger core parameter, e.g. $\gamma \sim 2$. This emphasizes the importance of a more realistic determination of this parameter, which was also recently stressed by Eaglesham et al. [12].

A second remark is that there are, nevertheless, a noticeable number of cases for which the critical thickness falls far beyond the theoretical predictions, outside experimental uncertainties, even if the simplifications inherent to the theoretical calculations are taken into account. Such discrepancies which also exist between measurements on a given structure, have generated conflicts as to the reliability of experimental methods in detecting the transition $[22,30,34]$. More likely, these difficulties lend support to the increasingly accepted idea that thermodynamic equilibrium is not always reached in actual sample preparations $[11,13,20,23,28,35$, 36], in particular because of the large Peierls stress in crystals of diamant structure. The strain accomodation process is then dominated by the activation energy for dislocation nucleation and propagation, resulting in metastable behaviour which makes the critical thickness strongly dependent on growth conditions. This is likely the reason why models based on nucleation mechanisms $[10,11,20]$ did succeed, in some cases, in explaining strong deviations from equilibrium, as shown in figure 5 for the People and Bean model [10].
In connexion with metastability and nucleation processes, it is worth mentioning the influence of the substrate growth area, recently stressed [36, 37], on the interface dislocation density. Luryi and Suhir [37] have theoretically demonstrated the possibility of eliminating misfit dislocations through reduction of the transverse sample dimension $\ell$. This offers interesting promise in the development of technologies to reduce dislocation effects.

In summary, a simplified theoretical approach has been proposed to describe the thermodynamic equilibrium between bulk strain and misfit dislocations in single heterostructures. Some of the adopted simplifying assumptions could be released in further refinements, e.g. by taking into account the three dimensionality, the finite thickness of the epilayers, and the detailed cristallographic structure of misfit dislocations. This should require, accordingly, a better knowledge of the core contribution. Once coherently compared with one another, all equilibrium theories turn out to yield almost identical results, so that the widespread agreement of Matthews' approach with a number of experiments is valid for all of them and, in particular, for the present theory, as well. Remaining discrepancies, on the other hand, have been admittedly ascribed to metastable behaviour. In fact, the occurrence of such a behaviour together with the role of lateral dimensions, are fairly attractive features since they allow us to foresee the possibility of removing strict limitations on allowed thicknesses of mismatched materials and, thereby, to produce structures free from misfit dislocations. To this end the present approach could help in a systematic evaluation of metastable contributions.

\section{Appendix I}

We have to calculate the numerical series

$$
Z=\sum_{n=1}^{\infty} \frac{\sin ^{2}(\pi n c / p)}{n^{3}}=\frac{1}{2} \sum_{n=1}^{\infty} \frac{1-\cos (2 \pi n c / p)}{n^{3}}
$$

Let $x=2 \pi c / p$. The series in $\cos x$ in the r.h.s. can be obtained by means of two integrations of the identity 38 .

$$
\sum_{n=1}^{\infty} \frac{\cos n x}{n}=-\ln 2-\ln \left(\sin \frac{1}{2} x\right)
$$

which gives

$$
Z=\frac{1}{2} \int_{0}^{x} \mathrm{~d} x^{\prime} \int_{0}^{x^{\prime}} \mathrm{d} x^{\prime \prime} \sum_{n=1}^{\infty} \frac{\cos \left(n x^{\prime \prime}\right)}{n}=-\frac{1}{2} \int_{0}^{x} \mathrm{~d} x^{\prime} \int_{0}^{x^{\prime}} \mathrm{d} x^{\prime \prime}\left[\ln 2+\ln \left(\sin \frac{1}{2} x^{\prime \prime}\right)\right] .
$$

The calculations are elementary and lead to

$$
Z=\frac{\pi^{2} c^{2}}{p^{2}}\left(\ln \frac{p}{2 \pi c}+\frac{3}{2}+\frac{\pi^{2} c^{2}}{36 p^{2}}+\cdots\right)
$$




\section{Appendix II}

The series $S(z)$ can be reduced to logarithmic series

$$
\begin{aligned}
S(z) & =\sum_{n=1}^{\infty} \frac{\sin \left(\frac{1}{2} k_{n} c\right)}{n} \mathrm{e}^{-k_{n} z} \\
& =\sum_{n=1}^{\infty} \frac{1}{2 i n}\left[\exp \left[-2 \pi n\left(z-\frac{1}{2} i c\right) / p\right]-\exp \left[-2 \pi n\left(z+\frac{1}{2} i c\right) / p\right]\right] \\
& =-\frac{1}{2 i} \ln \frac{1-\exp \left[-2 \pi\left(z-\frac{1}{2} i c\right) / p\right]}{1-\exp \left[-2 \pi\left(z+\frac{1}{2} i c\right) / p\right]}=\operatorname{Artg} \frac{\sin (\pi c / p)}{\exp (2 \pi z / p)-\cos (\pi c / p)} .
\end{aligned}
$$

Since $|z| \leqslant c \ll p$, this can be approximated by

$$
S(z) \cong \operatorname{Arctg} \frac{\pi c / p}{2 \pi z / p-\pi^{2} c^{2} / 2 p^{2}} \cong \operatorname{Arctg} \frac{c}{2 z}+\mathcal{O}(c / p) .
$$

Making use of $S(z)$ and its derivatives, the detailed expression of $W_{c}^{(\mathrm{el})}$, as given by (11), becomes, after the evident integration by $x$,

$$
\begin{aligned}
W_{\mathrm{c}}^{(\mathrm{el})}= & \frac{2 G a^{2}}{\pi^{2} c(1-\nu)^{2}} \int_{0}^{\mathrm{c} / 2}\left[(1-\nu)\left(\operatorname{Arctg} \frac{c}{2 z}-\frac{c z}{c^{2}+4 z^{2}}\right)^{2}+\frac{(1-\nu) c^{2} z^{2}}{\left(c^{2}+4 z^{2}\right)^{2}}\right. \\
& \left.-\nu\left(\operatorname{Arctg} \frac{c}{2 z}-\frac{c z}{c^{2}+4 z^{2}}\right) \frac{2 c z}{\left(c^{2}+4 z^{2}\right)}+\frac{\left(c^{2}-4 z^{2}\right)^{2} c^{4}}{6\left(c^{2}+4 z^{2}\right)^{4}}\right] \mathrm{d} z+\mathcal{O}(c / p) .
\end{aligned}
$$

Putting $x=2 z / c$, all integrations are easily performed and yield, to order $c / p$,

$$
\begin{aligned}
W_{\mathrm{c}}^{(\mathrm{el})}(0) & =\frac{G a^{2}}{4 \pi(1-\nu)^{2}}\left[(1-\nu)\left(\frac{\pi}{4}+3 \ln 2-\frac{4 C}{\pi}\right)-\frac{7}{24}-\frac{3}{2} \ln 2+\frac{36 C-7}{18 \pi}\right] \\
& =\frac{G a^{2}}{4 \pi(1-\nu)}\left(1.696-\frac{0.289}{1-\nu}\right),
\end{aligned}
$$

where $C=0.9159 \ldots$ is the Catalan constant [38].

\section{References}

[1] Herbaux C., Di Persio J. and Lefebvre A., Appl. Phys. Lett. 54 (1989) 1004.

[2] Chang K. H., Bhattacharya P. K. and Gibala R., J. Appl. Phys. 65 (1989) 3391.

[3] Nishioka T., Itoh Y., Sugo M., Yamamoto A. and Yamaguchi M., Jpn J. Appl. Phys. 27 (1988) L-2271.

[4] Yamaguchi M., Sugo M. and Itoh Y., Appl. Phys. Lett. 54 (1989) 2568.

[5] Tsai H. L. and MAtyi R. J., Appl. Phys. Lett. 55 (1989) 265.

[6] Van der Merwe J. H., Proc. Phys. Soc. London A $63(1950) 616$.

[7] VAn Der Merwe J. H., J. Appl. Phys. 34 (1963) $117 ; 34$ (1963) 123.

[8] VAN Der Merwe J. H., Surf. Sci. 31 (1972) 198.

[9] Matthews J. W. and Blakeslee A. E., J. Cryst. Growth 27 (1974) 118.
[10] People R. and Bean J. C., Appl. Phys. Lett. 47 (1985) $322 ; 49$ (1986) 229.

[11] Marée P. M., Barbour J. C., VAn der Veen J. F., Kavanagh K. L., Bulle-Lieuwa C. W. T. and Viegers M. P. A., J. Appl. Phys. 62 (1987) 4413.

[12] Eaglesham D. J., KvaM E. P., Maher D. M., Humphrey C. J. and Bean J. C., Philos. Mag. 59 (1989) 1059.

[13] Ji G., Ristic V. M. and Reddy U. K., Can. J. Phys. 67 (1989) 639.

[14] Olsen G. H. and Ettenberg M., J. Appl. Phys. 48 (1977) 2543.

[15] Zhe-Chuan Feng and Hong-Du LiU, J. Appl. Phys. 54 (1983) 83.

[16] Timoschenko, Theory of Elasticity (McGraw Hill, New York) 1934. 
[17] HiRTh J. P. and Lothe J., Theory of Dislocations, 2nd ed. (Wiley, New York) 1982.

[18] Louchet F. and Thibault-Dessaux J., Rev. Phys. Appl. 22 (1987) 207.

[19] Sugita Y., Tamura $M$. and Sugawara K., $J$. Appl. Phys. 40 (1969) 3089.

[20] Fiory A. T., Bean J. C., Feldman L. C. and RobiNSON I. K., J. Appl. Phys. 56 (1984) 1227.

[21] Nomura T., MAeda Y., MiYao M., MinORU HagiNo and Ishikawa K., Jpn J. Appl. Phys. 26 (1987) 908.

[22] Orders P. J. and Usher B. F., Appl. Phys. Lett. 50 (1987) 980.

[23] Marée P. M. J., Olthof R. I. J., Frenken J. W. M., VAN DER VEen J. F., BUlle-LieUWMA C. W. T., VIEGERS M.P. A. and ZALM P. C., J. Appl. Phys. 58 (1985) 3097.

[24] Anderson N. G., Laidig W. D., Kolbasd R. M. and Lo Y. C., J. Appl. Phys. 60 (1986) 2361.

[25] Anderson T. G., Chen Z. G., Kulakovskit V. D., Uddin A. and Vallin J. T., Appl. Phys. Lett. 51 (1987) 752.

[26] Reithm ̇̀ier J.-P. and Cerva H., Appl. Phys. Lett. 54 (1989) 48.

[27] Elman B., Koteles E. S., Melman P., Jagannath
C., LeE J. and Dugger D., Appl. Phys. Lett. 55 (1989) 1659.

[28] Weng S.-L., J. Appl. Phys. 66 (1989) 2217.

[29] Kasper E., Herzog H. J. and Kibbel H., Appl. Phys. 46 (1975) 89.

[30] Kohama Y., WATANabe Y. and FukUda Y., Jpn J. Appl. Phys. 2 (1987) L1944.

[31] Fritz I. J., Picraux S. T., Dawson L. R., DrumMOND T. J., LAIDIG W. D. and ANDERSON N. G., Appl. Phys. Lett. 46 (1985) 967.

[32] Fritz I. J., Gourley P. L. and Dawson L. R., Appl. Phys. Lett. 51 (1987) 1004.

[33] Houghton D. C., Gibbings C. J., Tuppen C. G., Lyons M. H. and Halliwell M. A. G., Appl. Phys. Lett. 56 (1990) 460.

[34] Woodhead J., Appl. Phys. Lett. 55 (1989) 2147.

[35] Hull R., Bean J. C., Werder D. J. and LeibenGUTH R. E., Appl. Phys. Lett. 52 (1988) 1605.

[36] Fitzgerald E. A., Watson G. P., Proano R. E. and Ast D. G., J. Appl. Phys. 65 (1989) 2220.

[37] LurYi S. and SuhIR E., Appl. Phys. Lett. 49 (1986) 140.

[38] Gradshteyn I. S. and Ryzhik I. M., Table of Integrals, Series and Products (Acad. Press, New York) 1965. 